\title{
FEA OF PCB MULTILAYER STACK UP HIGH VOLTAGE PLANAR TRANSFORMER FOR AEROSPACE APPLICATION
}

\author{
Kiran Gaikwad ${ }^{1}$; Tobias Bartusch ${ }^{2}$ \\ ${ }^{1}$ Analytical Instrument, Measurement and Sensor technology, \\ Hochschule Coburg, Germany \\ Kirang6644@gmail.com \\ ${ }^{2}$ Airbus Defence and Space, Ottobrunn, Germany \\ Tobias. Bartuscheastrium. eads. net
}

\begin{abstract}
High voltage planar transformer is a technology which can replace conventional transformer with its distinct advantages of saturation and cost efficiency. This paper includes, study and solution methods for PCB winding configuration in planar magnetic elements with multilayer stack up of PCB Cu-tracks, producing High voltage power supply for aerospace application. With finite element analysis (FEA) simulations, different simulation outcomes are discussed for inspecting flux intensity and current density distribution with computing Electric field strength and Magnetic fields.
\end{abstract}

In principal conclusion of study, complete analysis and some practical design guidelines for multilayer PCB stack up are discussed in this paper.

\section{KEYWORDS}

Planar transformer, PCB tracks, FEA.

\section{INTRODUCTION}

In recent years, industries such as military/aerospace, hybrid vehicles, and alternative energy are benefiting from planar technology that features high performance from a low-profile design package and are ideal for applications where the space, weight and cost are vital. This printed circuit replaces typically wire wound coils to flat, extremely durable, high temperature resilient, high inner layer, and voltage dielectric breakdown rating PCB tracks. Planar magnetics refer to high-frequency transformers and inductors exhibiting advantages in electrical, thermal and mechanical aspects [1].

With Planar technology, instead of constructing magnetics components as transformer and inductors of wire-wound assemblies on bobbin, the planar PCB magnetics take benefits from using PCB-tracks as windings. Main advantages are the simplification of assembly and no longer requirement of the magnetics screening tests. This is a part of the implemented cost reduction 
measures. Even layers with one and two layers are common. So it is also used in low voltage high current applications.

This paper presents optimized planar transformer solutions for aerospace application with low profile, EMI and low costs. It gives detailed studies influence of magnetic field distribution and eddy currents effects due to windings arrangements inside multilayer stack up. A finite element analysis simulation gives detailed calculation of field intensity and current density distribution of planar for aerospace application. Finally some design aspects and conclusions are discussed in this paper with necessary design guidelines.

\section{High Voltage APPLICATION}

High Power Amplifier (HPA) is needed in the satellite communication to operate transmission amplifiers. To supply electronics power, Electronic Power Conditioner (EPC) is used in addition to tube with the necessary operating voltages. It acts as a High Power Amplifier when connected together with a Klystron. The klystron is a vacuum tube that takes advantage of runtime of electrons to creation or reinforcement of high-frequency signals, while the disadvantage is heavy weight and large size power supply. Also a core equipment of an Ion Thruster Unit is the High Voltage Power Supply (HVPS), which is dedicated to condition the electrical power from the spacecraft main bus. Key issue of this power equipment is the generation of high voltage in the range of $1.2 \mathrm{kV}$ for Kaufmann-Type and RIT-Type ion engines and up to $13 \mathrm{kV}$ for Field Emission Type thrusters [2] [3]. Thus planar magnetics were considered for its power supply which was challenging part for high voltage of $10-12 \mathrm{KV}$.

Figure below gives schematic for transformer section of HVPS power supply. Planar transformer plays an important role in this power supply. It has one primary winding and three secondary windings.

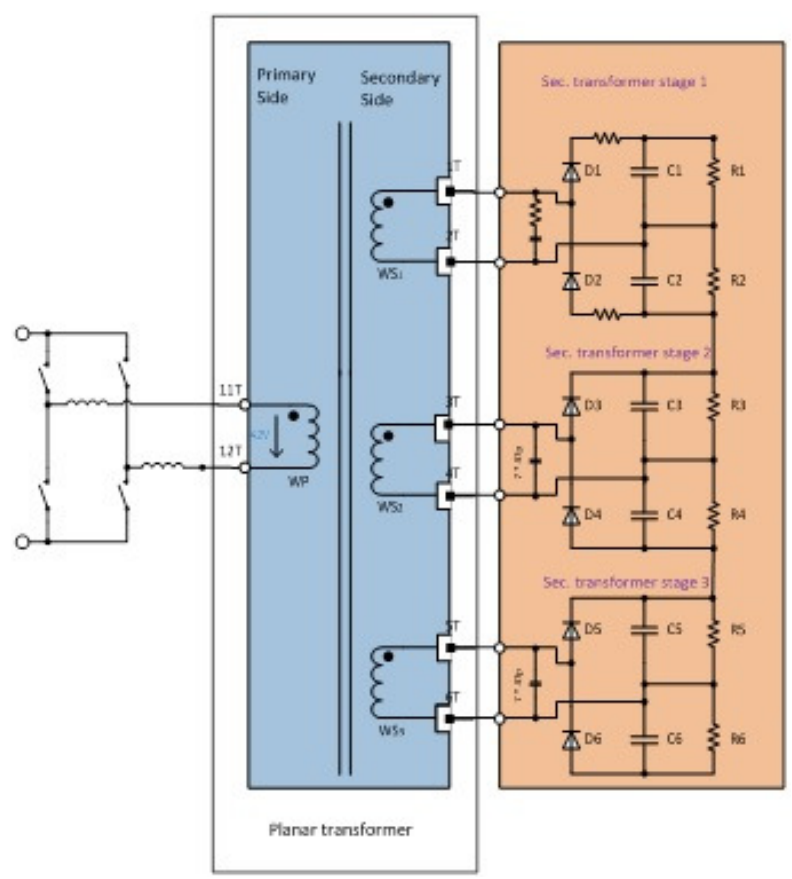

Figure 1. Schematic of Planar for high voltage application 
In electrical power transformer, there are primary, secondary and may be tertiary windings. Performance of a transformer mainly depends upon the flux linkages between these windings. For efficient flux linking between these windings, one low reluctance magnetic path common to all windings should be provided in the transformer. This low reluctance magnetic path in transformer is known as core of transformer. So selecting proper transformer core is one of the important and crucial parts in design of transformer.

After tradeoffs and market survey we concluded to select ferrite core for planar transformer importantly due to its high saturation and dimensions. This ferrite core by Magnetics ${ }^{\circledR}$ OR49938EC is of size $10 \times 20.3 \times 37.5 \mathrm{~mm}$, pair of E cores and manufactured from P\&R grade material. This material type is a low to medium frequency power material with minimum core losses at $100 \mathrm{kHz}, 100 \mathrm{mT}$ and $100^{\circ} \mathrm{C}$ for use of power transformers up to $500 \mathrm{kHz}$ [4]. This core has high permeability and low losses at high flux densities and temperatures. Also it has high saturation and low existing currents [5].

Figure 2 shows the winding arrangement of high voltage planar transformer. Primary windings has two turns $(\mathrm{WP}=2)$ and the secondary has 96 turns (WS1=WS2=WS3=96).

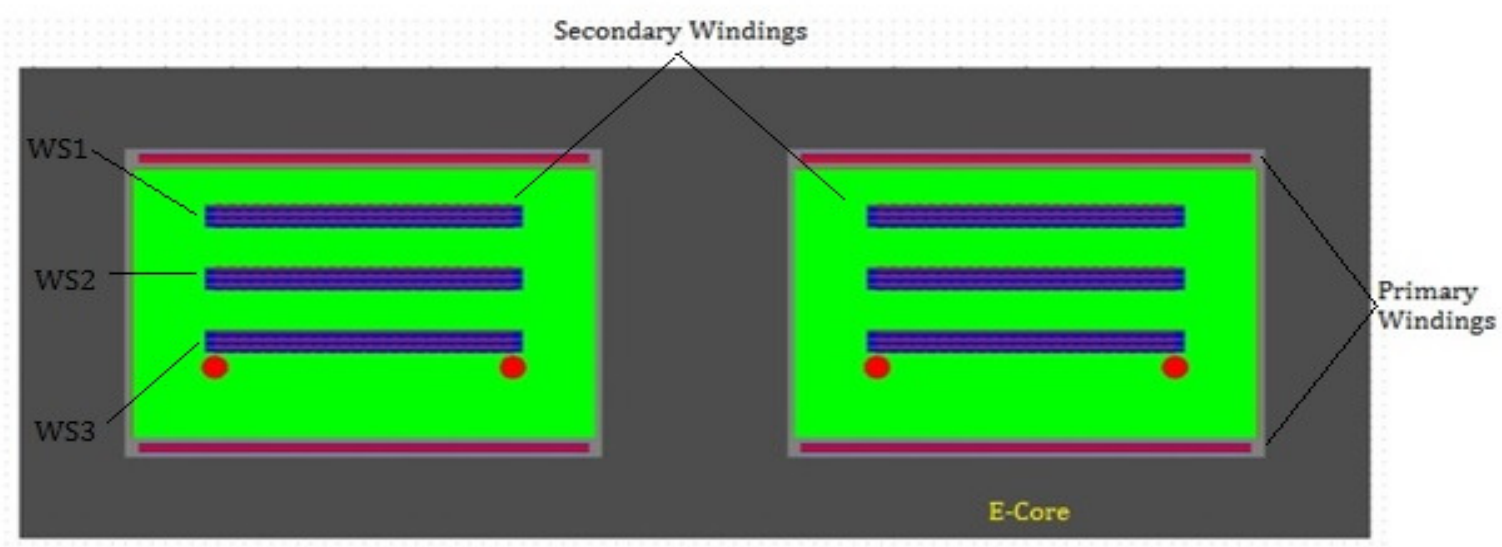

Figure 2. Winding arrangement of high voltage transformer

Figure shows PCB stack up implemented in E-Core. Details of boundary/sources are mention below.

- $\quad$ Total no of PCB stack-up : 3

- $\quad$ Primary no of turns $\mathrm{N}_{\mathrm{PRI}}: 2$

- $\quad$ Secondary no of turns $\mathrm{N}_{\mathrm{SEC}}: 288$

(Per layer: 24; one stack-up: 96)

- Primary Voltage : $51 \mathrm{~V}$

- Secondary voltages :

WS1: 0-3270 volt

Total windings: 96 ; distributed voltage is $\Delta \mathrm{U} 34.5$ volt per winding.

WS2: 3300-6770 volt

Total windings: 96 ; distributed voltage is $\Delta \mathrm{U} 36.5$ volt per winding. WS3: 6800-10360 volt

Total windings: 96 ; distributed voltage is $\Delta \mathrm{U} 37.5$ volt per winding. 


\section{STACK UP DESIGN}

Figure 3 shows one layer of PCB stack up design from secondary windings, which includes PCB tracks of $0.06 \mathrm{~mm}$, same size feedback and these layers are separated with FR4-epoxy core of $0.25 \mathrm{~mm}$. Feedback layer is used because of large voltage difference i.e. around 500 Volts between each layer, which increases losses and high electric field around windings. Implementation of this feedback layer reduces these effects. Highlighted area in Figure 3 shows single PCB track winding with its mechanical dimensions.

Width: $0.7 \mathrm{~mm}$ (down)

Width: $0.6 \mathrm{~mm}$ (up): (Size considered after effect of etching process)

Height: $0.06 \mathrm{~mm}$

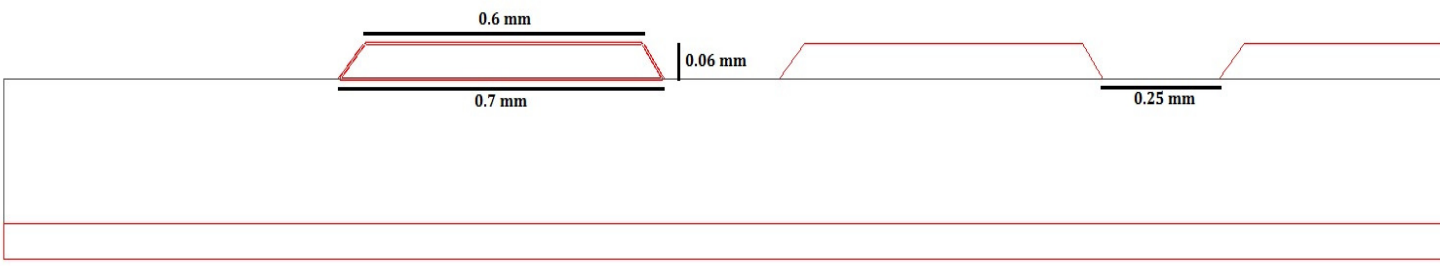

Figure 3. Single layer dimensional description

This design gave us small layer size of $0.37 \mathrm{~mm}$. We have a winding layer of $0.06 \mathrm{~mm}$ and further the feedback connection has the same geometry. These layers are separated with FR4-epoxy core of $0.25 \mathrm{~mm}$. Dimensions details are mention in the figure 4 .

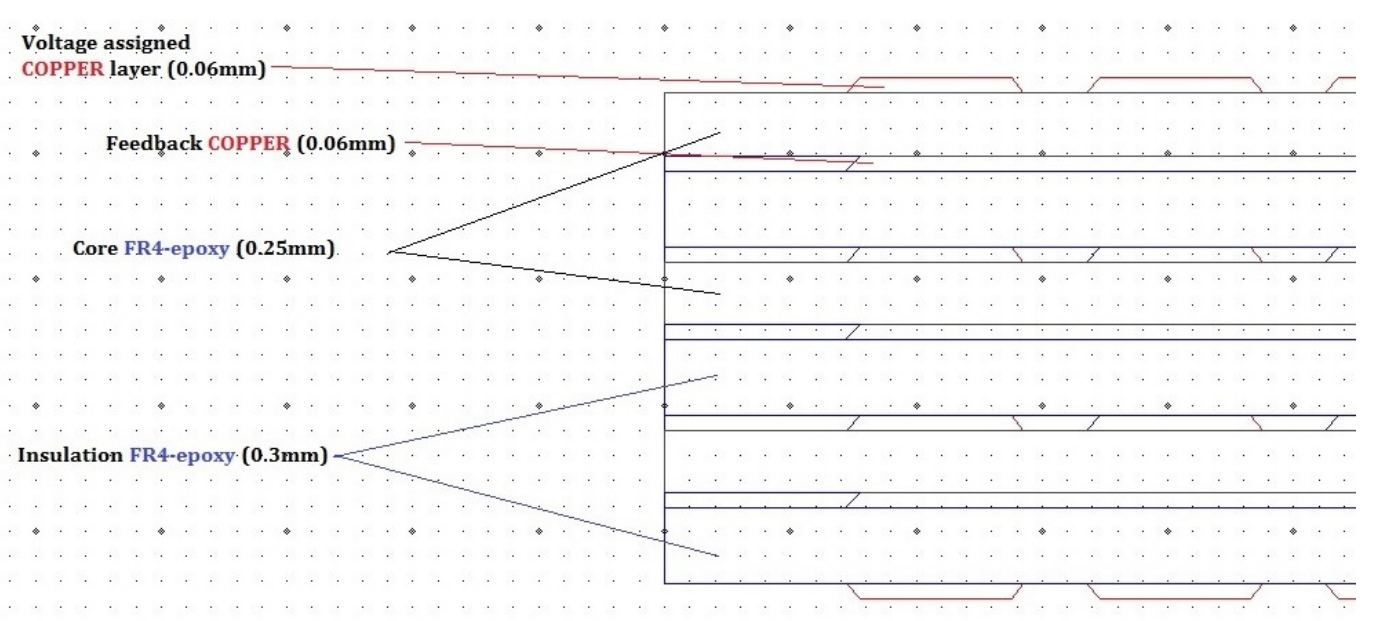

Figure 4. Complete PCB stack up for single secondary winding

In total 24 PCB copper tracks are used in one layer separated with distance of $0.25 \mathrm{~mm}$, so total available width is $24 \mathrm{~mm}$. After stacking single layer four times and separated with FR4-epoxy insulation of size $0.3 \mathrm{~mm}$, total height of stack-up is $2.07 \mathrm{~mm}$. Figure 5 shows final view of single stack up for one of the secondary winding with total no of 96 windings. Top view for windings structure is shown in Figure 6. These windings are made with curve shapes at edges which avoid sharp edges and corresponding losses. 
Figure 5. Final view of single stack up of secondary windings

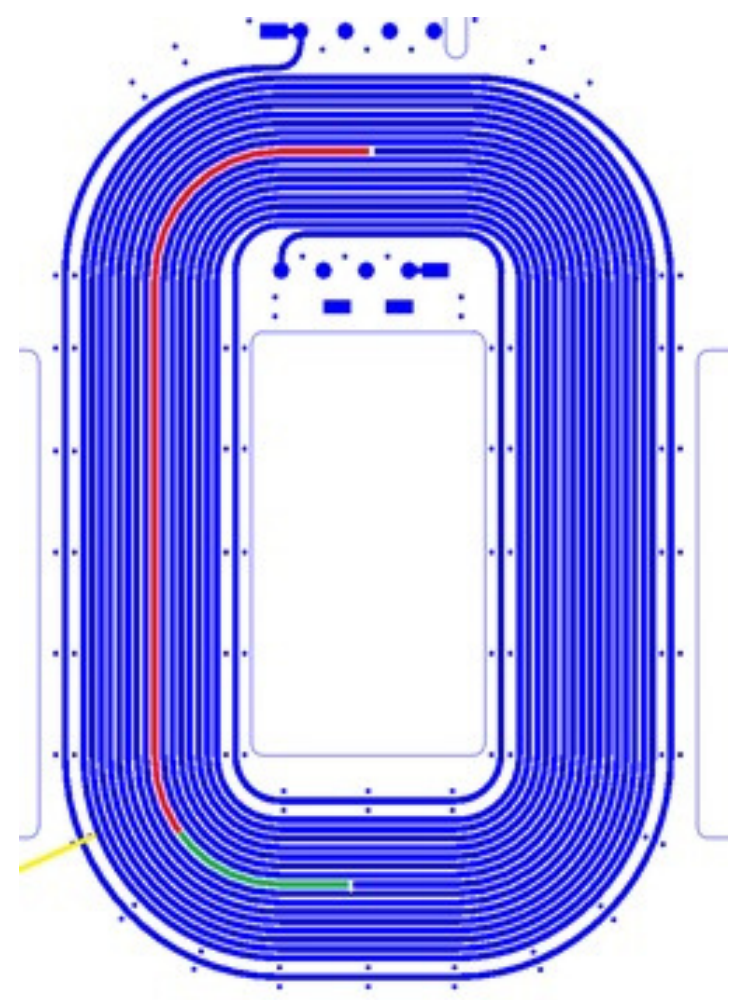

Figure 6. Top view of copper tracks in secondary

\section{Finite ELEMENT ANALYSIS (FEA)}

This study is conducted in Finite Element Analysis tool. This software is powerful and accurate for two-dimensional, electrostatic, electromechanical and thermal analysis. Its AC magnetic capability solves systems that have significant effects from induced eddy currents, skin effect and Proximity effect. Also we can calculate power loss, core loss, and impedance for frequency, force, torque, inductance and stored energy. Additionally flux lines, Flux density, current distribution and energy densities can be plotted. 
To analyze field distribution, geometrically data is implemented in this tool with necessary mechanical dimensions as mentioned earlier. Then after material properties and excitations for each copper track according to mentioned in boundary/source details are defined. By using a FEA tool we were able to calculate the electrostatic and magneto static fields, also creates finite element mesh. In our study we are interested in flux and current distribution, the magnetic intensity and electric-field intensity.

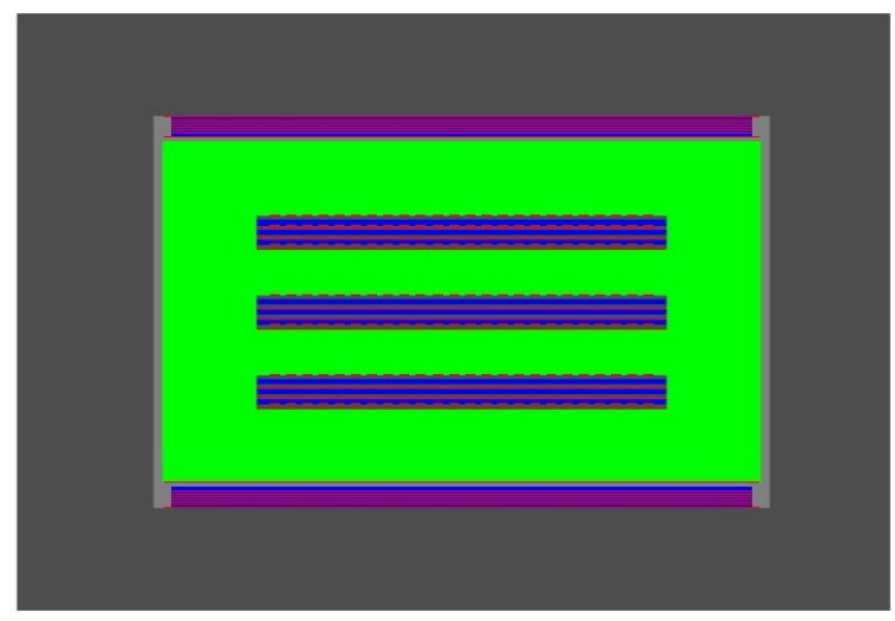

Figure 7. Calculation window of the planar transformer

Figure 7 shows window used for calculation and finite element analysis. In previous figures two extra round structures are shown, these are nothing but extra added copper wire coils which are connected to the last windings of the secondary. These structures were implemented after iterative optimization to minimize high electrostatic fields at higher electric potential windings. Finite element mesh for this geometry is shown in figure 8. This allows the software to compute field solution in each element. Smaller the element, more accurate is the final solution. In this case, the total numbers of triangles are round $10 \mathrm{k}$.

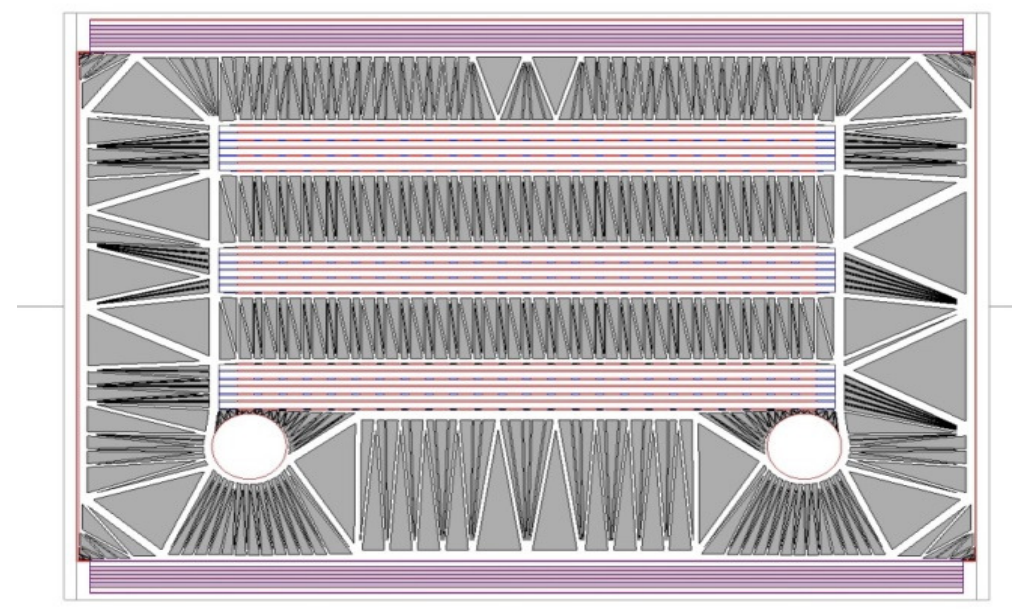

Figure 8. Mesh plotting of the calculation 


\subsection{Electrostatic Simulation}

Electric potential in different windings are distinctly different, resulting in higher potential. To keep the insulation between them we have arranged windings as WP-WS1-WS2-WS3-WP. This intersected winding scheme of primary and secondary together also helps to reduce effects like proximity effect.

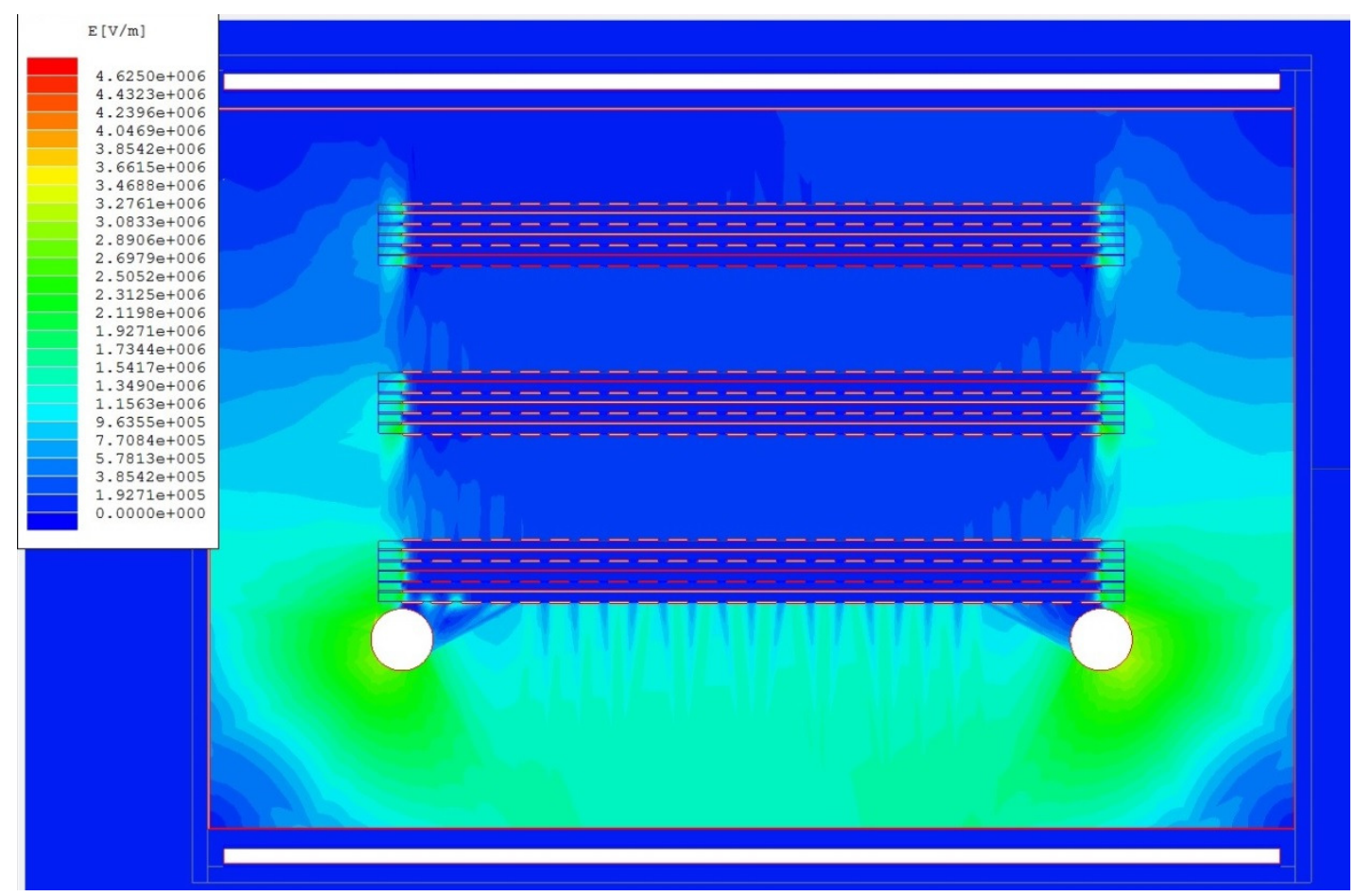

Figure 9. Electric field intensity (E) distribution of windings

Electric field intensity distribution of stack up is shown in figure 9. In this we can observe that the electrical field in WS3 and WS2 is comparatively more than the WP and WS1 due to high voltage assignment. FR-4 epoxy acts as perfect insulation between these winding stack up. Higher potential is at WS3, to reduce this different approaches were considered and finally came to conclusion with rings to the outer edge windings. As shown in Figure 9 two rings of $2 \mathrm{~mm}$ in diameter are constructed and assigned to the same voltage of edges winding in last stack up layer. This helped to reduce field strength in critical areas of the complete stack up. After implementing different sizes of rings, changing their position and distances good achievable value of electric field is $\mathrm{E}: 4.63 \mathrm{kV} / \mathrm{mm}$.

Different structures were in consideration to reduce this field strength in critical areas. Faradays cage was one of the important concepts. In this approach we implemented faradays cage around complete stack-up. This cage was of $0.25 \mathrm{~mm}$ thickness, consisting of copper and set to floating potential in simulation. Results after implementation of faradays cage were prominent and observed field strength was $1.10 \mathrm{kV} / \mathrm{mm}$ lesser than results from figure 9 . But it was hard to implement faradays cage around complete stack up and also was expensive to construct from manufacturing side. So this design was not selected for further process of transformer building. 


\subsection{Eddy currents}

In majority of conventional transformer designs, calculating the total power loss was limited to core and copper losses. In this high voltage transformer, multilayer planar design different losses are considered. Effects such as Skin effect and Proximity effect increase the resistance of a winding above the DC resistance value. These two effects can be assessed by calculating the eddy current.

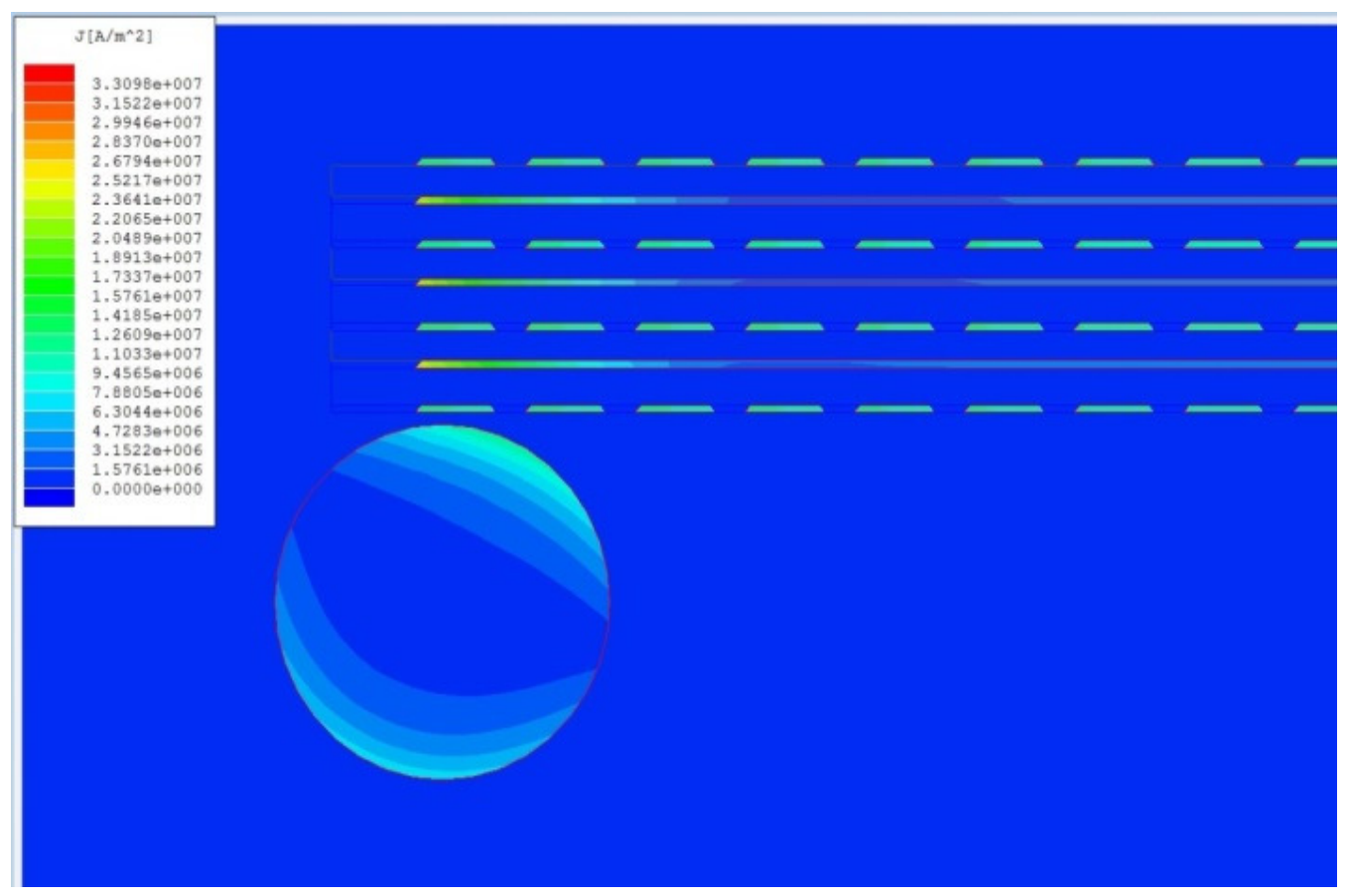

Figure: 10. Eddy currents distribution in windings

A common solution for reducing skin effect is to use round Litz wire [6]. Litz wire consists of many insulated strands that twist together generating a more uniform current distribution in the cross section of the conductor. As shown in design of winding structure we see this implementation, which means horizontally paralleling them. Another method to reduce the winding loss is to increase the cross-sectional area of the winding by vertically paralleling the windings, which means increasing the layers of PCB board [7].

Figure 10 shows eddy current distribution of windings. Eddy current is evenly distributed in the whole winding of the HV transformer. It agrees with the flux distribution mentioned in above section. Maximum eddy current density is present at the outer edges of the copper tracks of winding.

\section{DESIGN GUIDELINES}

Planar transformer is a growing field for the High voltage, high power transformers. Some different and important design guidelines are discussed below for proper high voltage planar transformer. Important guidelines are: 
- Secondary side windings are to be arranged according to the increase in voltage.

- It is better to implement intersected winding scheme of primary and secondary windings, together which helps to avoid effect as proximity effect.

- Proper shielding to be implemented in order to avoid high electric field in the windings. Sharp edges which are exposed to electrical field shall be avoided.

- Implementation of copper feedback track layer between two layers of voltage assignment.

- Proper insulation to be considered in different layers of stack up and between different windings of the secondary section to avoid voltage breakdown.

- Take the eddy current effect into account, the outer PCB track may be a little broader than the inner in order to reduce the AC resistance.

\section{CONCLUSION}

Planar magnetics is growing in the field of high voltage, high power transformer with large scope of application. Due to their higher reliability, better reproducibility and lower costs now are considerable option in aerospace application. Overall calculation results shows that it is possible to consider planar transformer with $\mathrm{PCB}$ tracks for high voltage high power aerospace application.

\section{REFERENCES}

[1] Quirke, M.T.; Barrett, J.J.; Hayes, M., "Planar magnetic component technology-a review,"Components, Hybrids, and Manufacturing Technology, IEEE Transactions on, vol.15, no.5, pp.884-892, Oct 1992

[2] Frank, H.; Franke, A.; Kiewe, B., "Single-stage high voltage power supply for ion engines", $8^{\text {th }}$ European Space Power Conference, Constance, Germany, 14-19 September 2008

[3] Michael, B.; Frank, H.; Kurt, R.; Matthias, G., "Generic high voltage power module for electrical propulsion", $29^{\text {th }}$ International electrical propulsion conference, Princeton university. Oct 31- Nov 4 2005

[4] Document OR49938EC Datasheet: OR49938EC Magnetics® planar E core.

[5] www.mag-inc.com

[6] Shen Wang; de Rooij, M.A.; Odendaal, W.G.; van Wyk, J.D.; Boroyevich, D., "Reduction of highfrequency conduction losses using a planar litz structure," Power Electronics, IEEE Transactions on, vol.20, no.2, pp. 261-267, March 2005

[7] Wei Chen; Yipeng Yan; Yuequan Hu; Qing Lu, "Model and design of PCB parallel winding for planar transformer," Magnetics, IEEE Transactions on , vol.39, no.5, pp. 3202-3204, Sept. 2003

\section{AUTHORS}

Kiran Gaikwad completed his Diploma and Bachelors in Electronics and Telecommunication engineering from university of Pune, India in year 2008 and 2011 respectively. Currently he is pursuing M.Eng in Analytical instrument, Measurements and Sensor technology from Hochschule Coburg (Coburg university of applied science), Coburg, Germany, in corporation with University of Shanghai for science and Technology (USST) Shanghai, China. from september 2012.

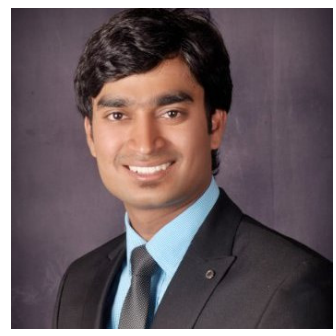


Electrical and Electronics Engineering: An International Journal (ELELIJ) Vol 3, No 4, November 2014

He was research assistant in Rössel messtechnik and summer intern in Airbus Defence and Space in electronic department for Space during Nov 2013- Jul 2014. He is currently doing his master thesis with Anode current measurement unit (ACMU) in Secondary power source of Electronic department Airbus Defence and Space, Friedrichshafen, Germany.

Tobias Bartusch is an expert with a Diploma in Telecommunications Electronic Engineering and Solid State Experimental Physics. His expertise is mainly in the fields of analog electronics, low noise circuits, vacuum electronics, RF systems, EMC, high voltage systems, electrical propulsion and plasma physics. In the years 2003 - 2008 he invented and developed a electrodeless plasma thruster and some applications for RF high voltage plasma and plasma cutting systems. Since end of 2008, he is at Airbus Defence and Space. He developed parts of the Galileo CMCU and actually, he is Systems Engineer for the IRNSS Atomic clock monitoring unit (ACMU) / RAFS project. Additionally he is a co engineer for the

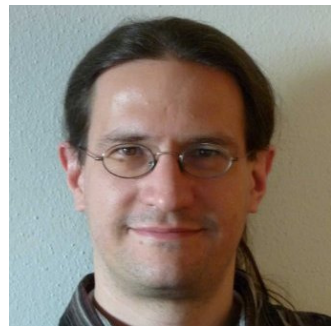
study of the high voltage planar transformer. 